\title{
Voltage regulation in distribution networks connected to PV sources
}

\author{
AlirezaSoltaniYas, AmenehSoltaniYas \\ MSc student - power engineer \\ Electrical engineer
}

\begin{abstract}
During the recent years, the renewable energy systems such as photovoltaic system have been raised widely. Voltage deviation because of the voltage fluctuations and also the unbalanced voltage due to PV counted as the serious problems in these systems. For reduction of this problems, the hybrid dynamic voltage regulator (C-DVR), consists of stage voltage regulator and AC chopper, is one of the effective devices. C-DVR can regulate the linear voltage continuously and rapidly. This article shows the C-DVR effect on numerical simulation.
\end{abstract}

Keywords: dynamic voltage regulator, AC Chopper, Photovoltaic system, Voltage control

\section{Introduction}

During the recent years, the distributed energy generation systems (DEG) such as photovoltaic system (PVs) and Fuel cell provide (FC) developed in a wide range.Most of DEGs connected via a single-phase power conditioner system (PCS). Because the most of unbalance currents relate to distribution line, the unbalance voltage may be bigger. In other word, the most of distribution systems have the radiation system (figure 1). For keeping the enough area, the distribution voltage line via a LRT in Substation (S/S) and SVR in a line via a polar transporter.

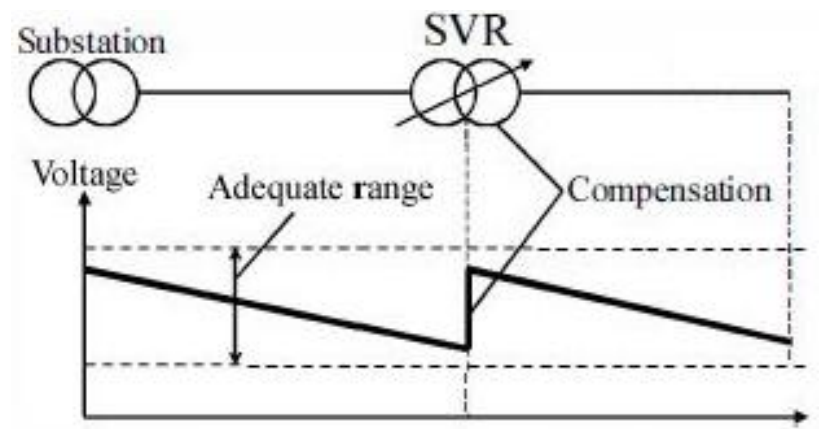

Fig1: radial distribution system

Since the LRT and SVR are transformers that control the voltage automatically, the voltage can change distinctly. Therefore, the unbalanced voltage in distribution system cannot set by these devices. On the other hand, the PV output oscillations depend on climate situations and the unbalanced voltage amount can change periodically or randomly. Therefore, the single-phase PV system may cause the one or two-step departure than the correct area. However, LRT and SVR have the low efficiency performance and cannot set the voltage effectually. In addition, the SVR performance can cause the friction and erosion. The high voltage setting devices presented such as Static Var Compensator (SVC) and the UPFC. These devices were expensive and their installation on production line is difficult. The TVR kind also presented as a more rapid voltage device but it cannot justifies the voltage continuously. This article presents a rapid voltage regulator that called hybrid dynamic regulator (C-DVR). C-DVR consists of step voltage regulator with thyristor (TVR configuration) and AC Chopper with IGBT. In this article, the C-DVR effect on prevention of voltage oscillation because of PV solar cells presence will be presented.

\section{C-DVR Configuration}

C-DVR consists of TVR and AC chopper and regulates the voltage via neutral voltage injection in distribution line series (fig2). Vsi ( $\mathrm{i}=\mathrm{u}, \mathrm{v}, \mathrm{w})$ is the source voltage, $\mathrm{R}$ and $\mathrm{L}$ are the resistance and 
Inductance of distribution line respectively. Vf is equal to neutral voltage that produced by C-DVR. Vs is the voltage of source side and $\mathrm{Vl}$ is the voltage on loading side.

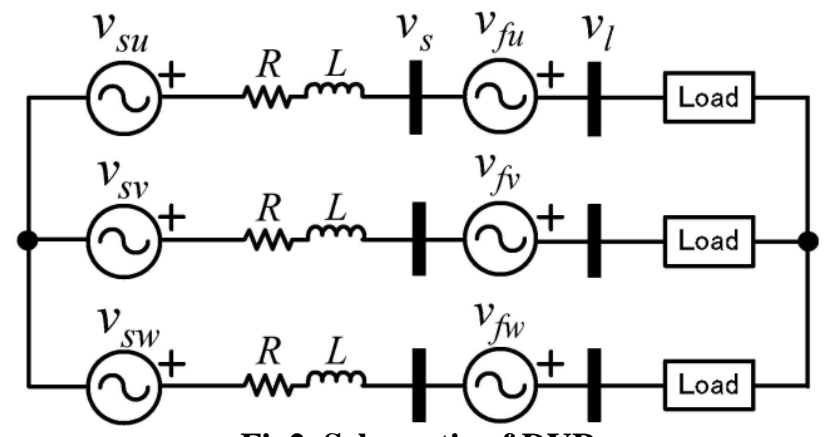

Fig2. Schematic of DVR

\section{Coupled AC chopper (CACC)}

C-DVR for triple-phase line consists of two AC chopper (figure 3). The CACC is back-boost and consists of five bi-side GTO (Th0 to Th4) and two bi-side IGBT. In addition to L-C filters consists of $\mathrm{Lf}$ and $\mathrm{Cf}$ at input side, $\mathrm{LO}$, and $\mathrm{C} 0$ at output side. GTOs are used in four level, regulating the voltage (figure 4). When the output voltage be zero, Th0 will be on. After the isolated voltage regulation by the GTOs, the AC chopper with $\mathrm{D}$ control duty in each area of $\mathrm{V}_{\text {RANGE }}$ regulatesthe output voltage precisely.

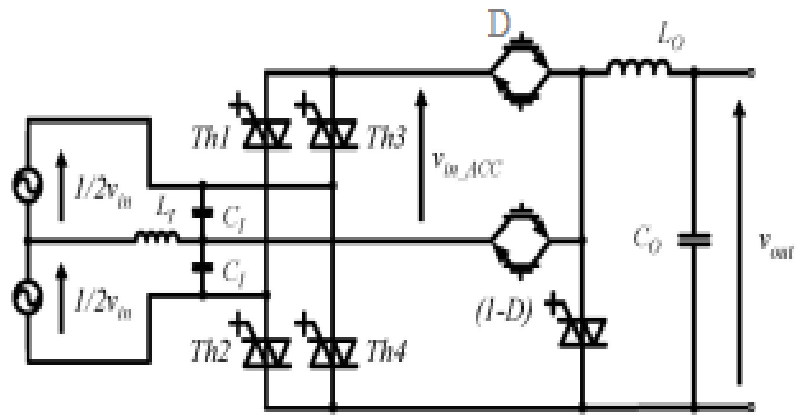

Fig3. The CACC structure

Figure 5 shows the current diagram in $\mathrm{V}_{\text {RANGE }}$ area. $\mathrm{V}_{\text {out }}$ for $\mathrm{V}_{\mathrm{PH}}$ obtained by equation 1:

VPH mode: $0.5 \mathrm{~V}_{\text {in }} \leq$ Vin- $\mathrm{ACC} \leq \mathrm{Vin}$ $\mathrm{V}($ out $)=((1+\mathrm{D}) / 2) \times \mathrm{Vinsin}(\mathrm{wt}) 1$

Figure 5a, shows the current change point lines by the IGBT current substitution. Therefore, HACC can have positive and negative output voltage continuously in $\mathrm{V}_{\text {in }}-0.5 \mathrm{~V}_{\text {in }}$ range. The other methods are same as $\mathrm{V}_{\mathrm{PH}}$ method.

VPL mode : $0 \leq \mathrm{Vin}-\mathrm{ACC} \leq 0.5 \mathrm{Vin} V($ out $)=((1-\mathrm{D}) / 2) \times \mathrm{Vin} \times \sin (\mathrm{wt}) \quad 2$

VNLmode: -0.5 Vin $\leq$ Vin- $A C C \leq 0$

VNHmode:-Vin $\leq$ Vin-ACC $\leq-0.5$ Vin
$\mathrm{V}($ out $)=((1-\mathrm{D}) / 2) \times \mathrm{Vin} \times \sin (\mathrm{wt})$

$\mathrm{V}($ out $)=((1+\mathrm{D}) / 2) \times \mathrm{Vin} \times \sin (\mathrm{wt})$
3

4 


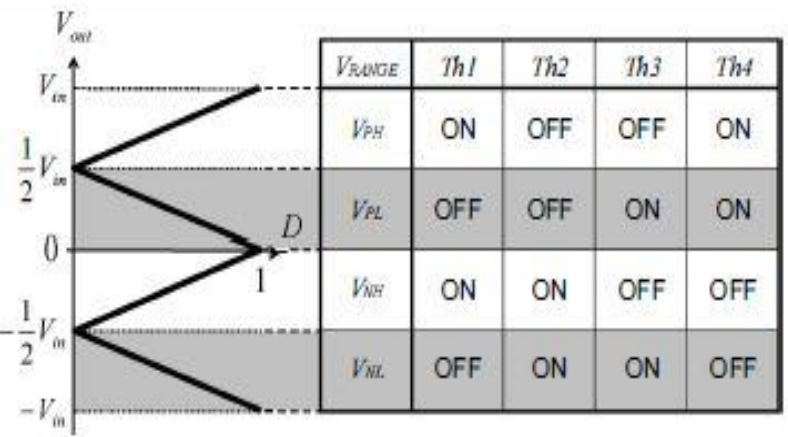

Fig4. CACC output

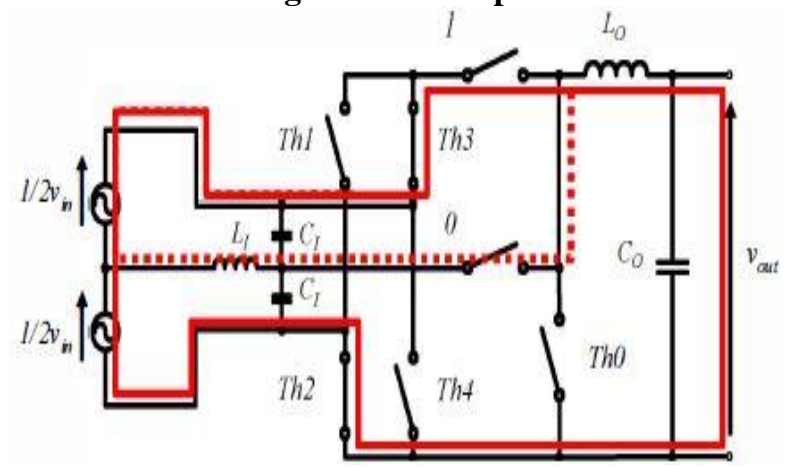

PH mode

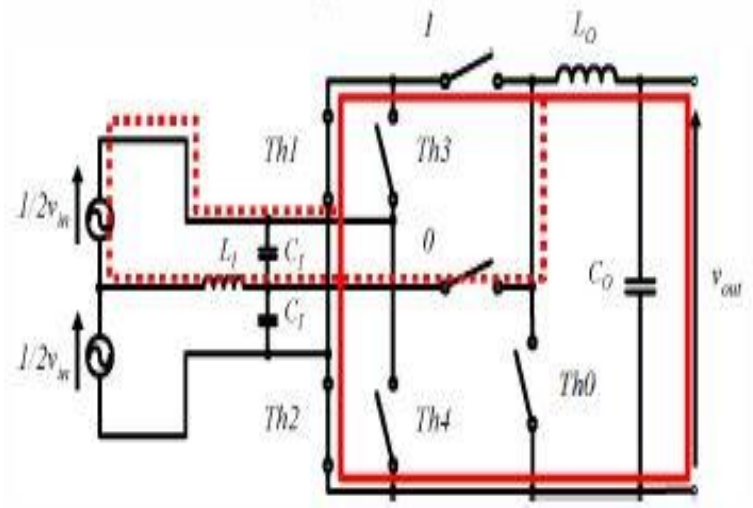

PL mode

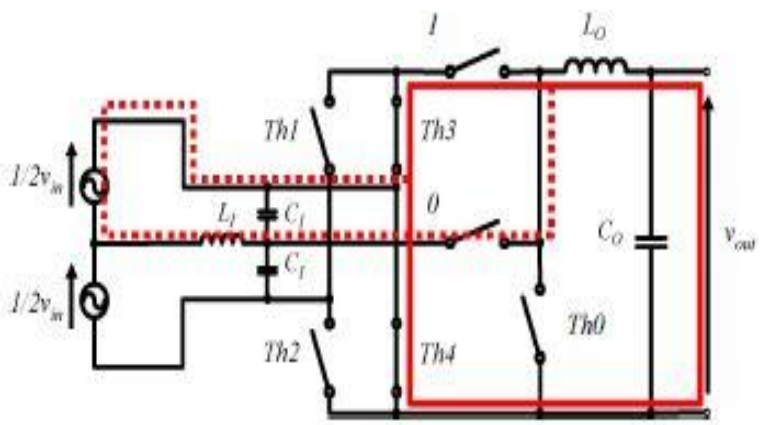

NL mode 


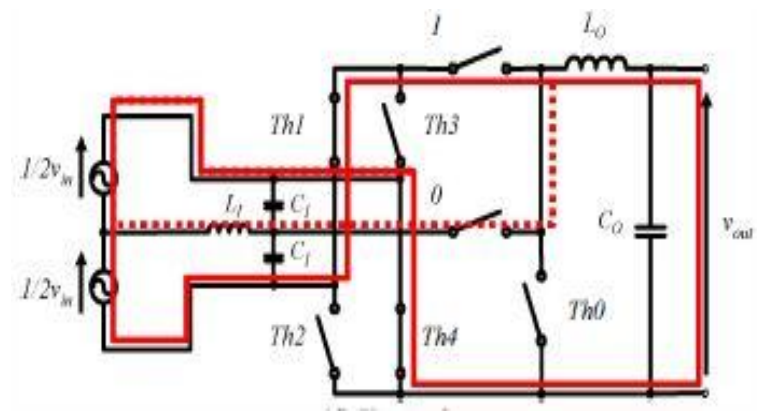

NH mode

Fig5. CACC output voltages

Considering the equations 1 to 4 and using of equation 5 , the $\mathrm{V}_{\text {in }}$ is determined and C-DVR can control the output voltage, determining the $\mathrm{K}$ coefficient.

$\mathrm{V}($ out $)=\mathrm{K} \times \mathrm{Vin}$

$(-1 \leq \mathrm{K} \leq 1)$

5

\section{C-DVR structure}

C-DVR structure has been presented in figure $6 . \mathrm{V}_{\mathrm{s}}$ and $\mathrm{V}_{\mathrm{l}}$ are the source and load sidevoltages respectively. C-DVR has two CACC for three-phase control. Each CACC input voltage provided via the line paralleling with transformer distribution line and CACC output voltage provided via the series transformers with distribution line. It should be considered because of the switch numbers, two CACC by the V connection.CACC can control the voltage continuously and rapidly.C-DVR can stop the voltage oscillations precisely.Therefore the enormous voltage oscillations caused by the big amount PV, can be neutral using the C-DVR.Reference voltage $\left(\mathrm{V}_{\text {ref }}\right)$ determined based on distribution line situations. Then the difference between $\mathrm{V}_{1}$ and $\mathrm{V}_{\text {ref }}$ will be calculated and neutral voltage is determined.

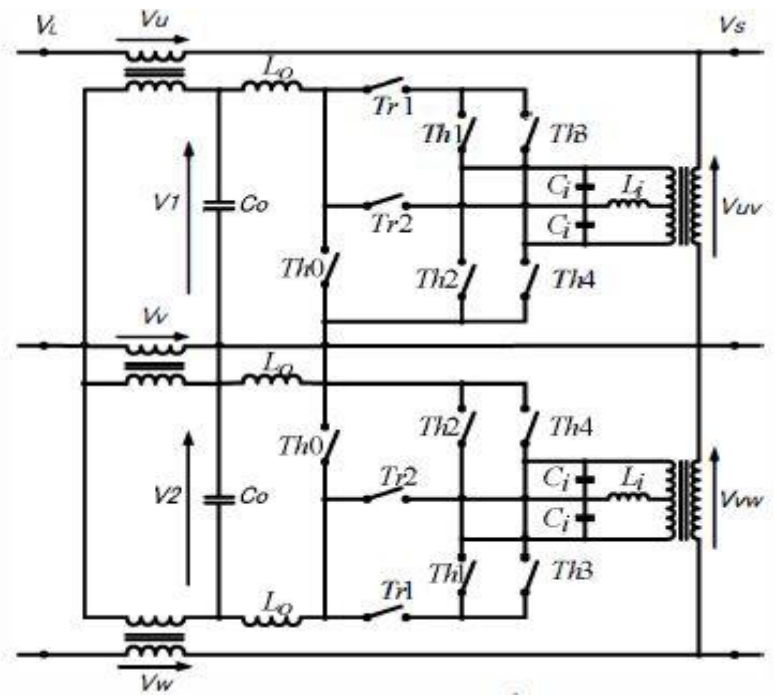

Fig6. The C-DVR structure

\section{The unbalance voltage neutralizing by C-DVR}

When the huge amount of PVs remain, they connect to the line separately, and then the unbalance voltage problem will be more serious. Unbalance voltage causes the problems for inductive engines.Therefore, the unbalance voltage stopping is one of the most important problems in power quality domain.

Magnitude and angle of output voltage is controlled by the input voltage $V_{\text {in }}$ which is the source line voltage $\left(\mathrm{V}_{\mathrm{s}}\right)$. For this aim, the CACC output voltage balanced using the second harmonic. The input voltage $\left(\mathrm{V}_{\text {in }}\right)$ expressed as equation 6 :

$\operatorname{Vin}=\operatorname{Vin} \times \sin \left(w t t_{\odot}\right)$

6

The reference of CACC $\mathrm{V}_{1}$ output voltage is determined by balance coefficient $(\mathrm{K})$ :

$\mathrm{V} 1=\mathrm{K} \times \mathrm{Vin} 7$

$\mathrm{K}=\mathrm{K} 0+\mathrm{K} 2 \times \sin \left(\mathrm{wt}++_{\varphi}\right)$ 
K0= Base Harmonic Coefficient, K2= Second Harmonic Coefficient

$\mathrm{V} 1=\mathrm{Vu}-\mathrm{Vv} 8$

$\mathrm{V} 2=\mathrm{Vv}-\mathrm{Vw}$

Output voltageV1=CACC1

$\mathrm{V} 2=$ CACC2output voltage

\section{Prevention of voltage oscillations}

\section{Simulation}

Approving the C-DVR effect, numerical simulation has been done, using the MATLAB software. The distribution model has been shown in figure 7. The huge amount of PV will be connected at the end of line. The maximum PV output is equal to four MW and as it can be seen in figure 8, has oscillation. C-DVR installed around the line center. C-DVR capacity equals to $180 \mathrm{KW}$, which can control the \pm 300 line voltage in stable state.

Linked loads regulated as R-L and its capacity equals to $125 \mathrm{Kva}$. In addition, the force factor is 0.98 . The other circumstances of simulation presented in table 1 . The C-DVR properties presented in table 2. The maximum output voltage $\left(\mathrm{V}_{\text {out-Max }}\right)$ limited by the distribution voltage line in C-DVR transform series.

When the effective amount of voltage in point $A$ equals to $V_{t}$, the maximum output voltage of C-DVR obtained, using the rotation of transformer direction and series' transformer ratio via the equation 9:

$\mathrm{V}($ out-max $)=\mathrm{Vi} \times(2000 / 660) \times(100 / 500)$

The output voltage $f$ each $\mathrm{CACC}$, regulated by the balance coefficient $(\mathrm{K})$ obtained from the equation 5 . Prevention the voltage oscillation caused by PV, K obtained from equation 10.

$\mathrm{K}=(\mathrm{Vi}-\mathrm{Vref}) /(\mathrm{Vi} \times(2000 / 660) \times(100 / 500))$

10

The reference voltage determined, considering the distribution line circumstances. Insimulation the $\mathrm{V}_{\text {ref }}$ regulated at $6340 \mathrm{~V}$. Simulation results are obvious in figure 9. Considering the voltage oscillation at point A, because of PV output oscillation, the voltage at point B is stopped enough, using the C-DVR.

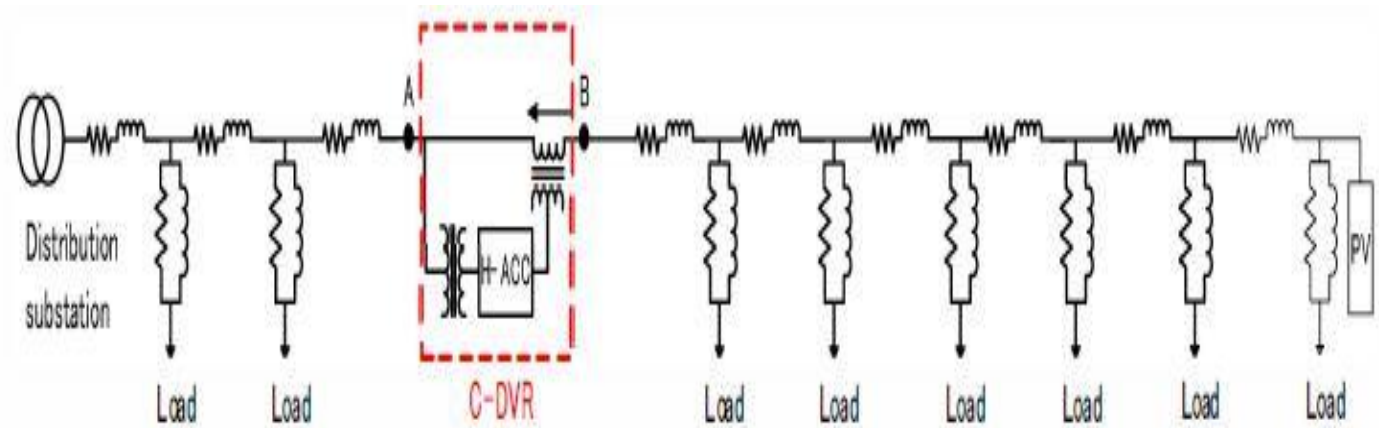

Fig7. Simulation model for voltage oscillation prevention

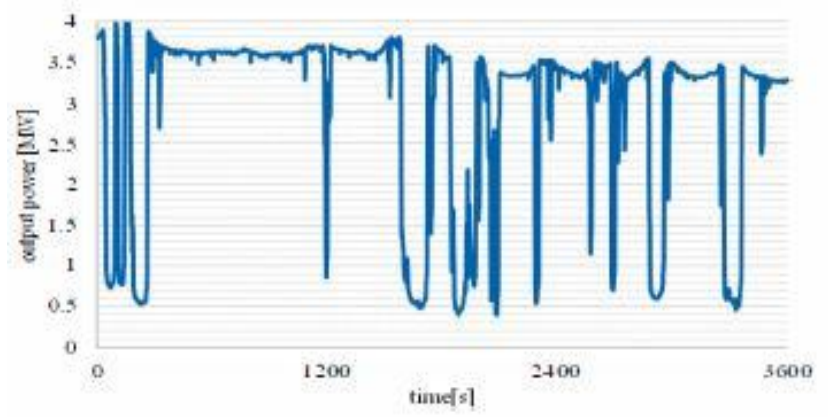

Fig8. PV output 


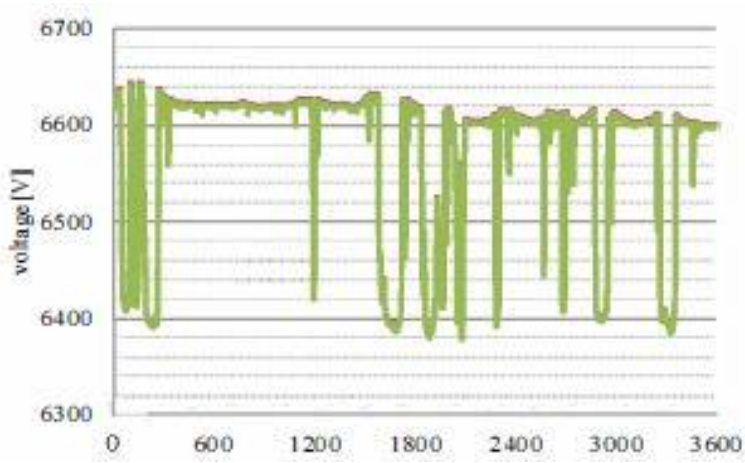

Voltage of Point A

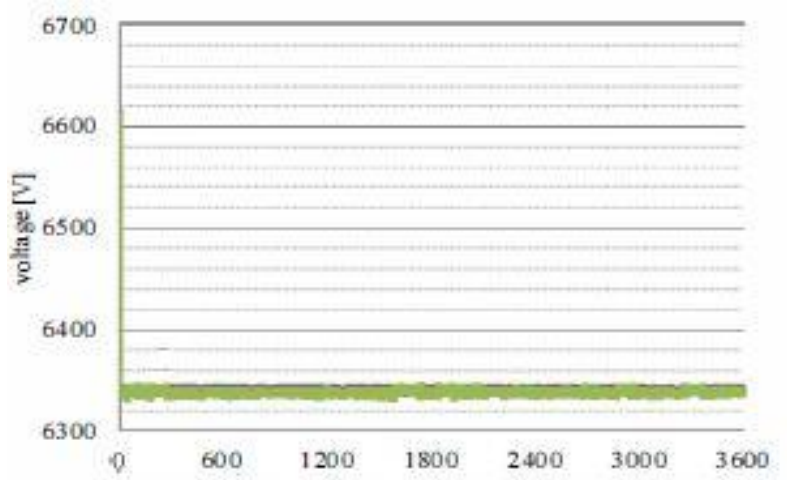

Voltage of Point B

Fig9. Simulation model for voltage oscillation prevention

\section{Unbalance Voltage Reduction}

In simulation, the PV did not use, showing the stopping efficiency of unbalance voltage.Simulation model is shown in figure 10. In this model, the sent voltage of substation $(\mathrm{S} / \mathrm{S})$ is unbalance. The magnitude of positive and negative periods in sent voltage are 6600 and $100 \mathrm{~V}$ respectively. In this case, the voltage unbalance ratio is $1.85 \%$.The $\mathrm{K} 0$ and $\mathrm{K} 2$ balance coefficient in equation 8 , determined zero.

The voltage simulation results at point B have been shown in figure 11. C-DVR at $0.05 \mathrm{~s}$ stops. After the negative period stopping by C-DVR, the unbalance voltage at point B reduces enough and constantly. The unbalance voltage ratio at point $\mathrm{B}$ equals to $0.08 \%$.

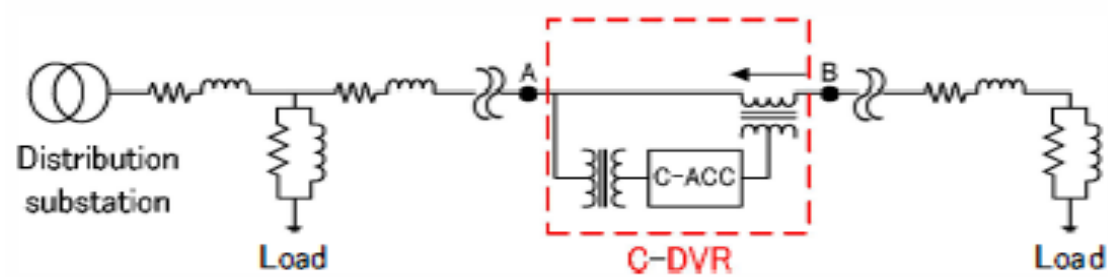

Fig10. Linear model simulation of unbalance voltage

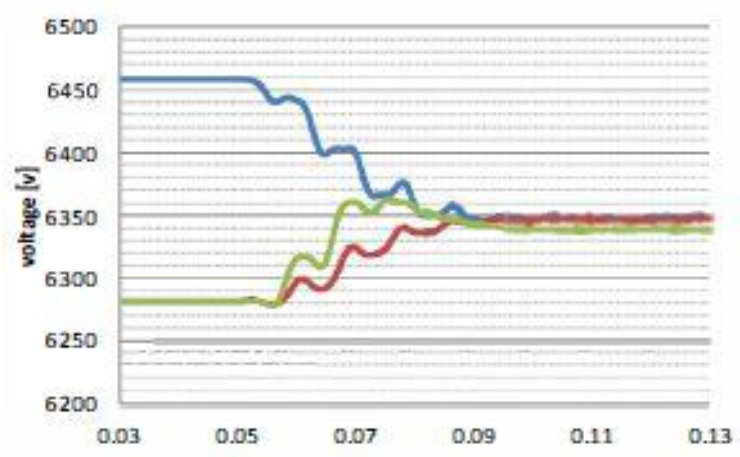

Fig11. Simulation results of unbalance voltage reduction 


\section{Conclusion}

In present study, a novel voltage regulator introduced, called C-DVR. C-DVR consists of stage voltage regulator with Thyristor (TVR configuration) and AC chopper by IGBT. It controls the voltage, neutral voltage injection to distribution line. The high speed and low cost are the C-DVR properties, comparing the SVC or UPFC.Via the numerical simulation and using the distribution model, it approved that C-DVR can stop the PV voltage oscillation. In addition, the unbalance voltage stopping control design has been presented. Unbalance voltage, is the sent voltage $(\mathrm{S} / \mathrm{S})$ that reduced via $\mathrm{C}-\mathrm{DVR}$ at loading point.

\section{References}

[1]. T.Kondo,J.Baba,andA.Yokoyama:"VoltageControlofDistributionNetworkwithaLargePenetrationofPhotovoltaic GenerationsusingFACTS Devices", IEEJTrans.PE,Vol.126,No.3,pp347-358,2007.

[2]. Y.Sasaki:"HighSpeedTVRforPowerDistributionLinesandTestResults",IEEJTrans.PE,Vo1.123,No.9,pplI051111,2009.

[3]. DivanM,J.Sastry:"VoltageSynthesisUsingDualVirtualQuadratureSourcesAnewConceptinACPowerConversion",PowerElectronics,I EEETransactions,Vol.23,pp3004-3013,2009.

[4]. DeepakDivan:"DesignandTestingofaMediumVoltageControllableNetworkTransformer Prototype with an IntegratedHybrid Active Filter", IEEE, pp4035-4042, 2010.

[5]. DebrupDas, Deepak Divan: "Power Flow Control in NetworksUsing Controllable Network Transformers", ICEE 2009, pp2224$2231,2011$.

[6]. Deepak Divan, JyotiSastry: "Control of Multilevel Direct ACConverters", ICEE, pp3077-3084, 2002012

[7]. Hirofumi Akagi, RyoheiKitada: "Control and DesignofaModular Multilevel Cascade BTB System Using BidirectionalIsolated DC/DC Converters' 